\title{
Activity/rest cycle and disturbances of structural backbone of cerebral networks in aging
}

\author{
Marion Baillet ${ }^{\mathrm{a}, \mathrm{b}, *}$, Bixente Dilharreguy ${ }^{\mathrm{a}, \mathrm{b}}$, Karine Pérès ${ }^{\mathrm{c}, \mathrm{d}}$, Jean-François Dartigues ${ }^{\mathrm{c}, \mathrm{d}}$, \\ Willy Mayo $^{\mathrm{a}, \mathrm{b}}$, Gwenaëlle Catheline $\mathrm{a}^{\mathrm{a}, \mathrm{b}, \mathrm{e}}$ \\ a Université de Bordeaux, INCIA, UMR 5287 - équipe NeuroImagerie et Cognition Humaine, F-33000 Bordeaux, France \\ ${ }^{\mathrm{b}}$ CNRS, INCIA, UMR 5287 - équipe NeuroImagerie et Cognition Humaine, F-33000 Bordeaux, France \\ ${ }^{\mathrm{c}}$ Université de Bordeaux, ISPED, Centre INSERM U1219 - Bordeaux Population Health Research Center, F-33000 Bordeaux, France \\ d INSERM, ISPED, Centre INSERM U1219 - Bordeaux Population Heath Research Center, F-33000 Bordeaux, France \\ e EPHE, PSL Research University, F-33000 Bordeaux, France
}

\section{A R T I C L E I N F O}

\section{Article history:}

Received 30 May 2016

Accepted 20 September 2016

Available online 21 September 2016

Keywords:

Aging

Diffusion tensor Imaging

Activity/rest cycle

Actigraphy

\begin{abstract}
A B S T R A C T
Objective: Although aging is associated with alterations of both activity/rest cycle and brain structure, few studies have evaluated associations between these processes. The aim of this study was to examine relationship between activity/rest cycle quality and brain structural integrity in aging subjects by exploring both grey and white matter compartments.

Material and methods: Fifty-eight elderly subjects ( $76 \pm 0.5$ years; $41 \%$ female) without dementia, sleep disorders and medications were included in the analysis. Actigraphy was used to measure parameters of activity/rest cycle (24-h amplitude, 24-h fragmentation and 24-h stability) and sleep (total sleep time and sleep fragmentation) over a minimal period of 5 days. Whole brain linear regression analyses were performed on grey matter volumes maps using voxel based morphometry and on white matter integrity using tract based statistics analyses.

Results: A lower 24-h amplitude and a higher sleep fragmentation were independently associated with a reduction of white matter integrity in models including age and gender as covariates. The association between 24-h amplitude and white matter integrity decreased but remained significant in a model accounted for sleep fragmentation, indicating a specific effect of 24-h cycle disturbances. No association with grey matter volumes was observed.

Conclusion: In elderly, not only sleep but also 24-h cycle disturbances were associated with altered structural connectivity. This alteration of structural backbone networks related to activity/rest cycle disturbances in aging might constitute a cerebral frailty factor for the development of cognitive impairment.
\end{abstract}

(c) 2016 Elsevier Inc. All rights reserved.

\section{Introduction}

Knowledge on activity/rest cycle and sleep disturbances in aging mainly results from subjective self-reported questionnaires (Buysse et al., 1989; Johns, 1991), easily applied in large epidemiologic or cohort-based investigations. However, these questionnaires are subject to the influence of perception and several studies have illustrated their relative inaccuracy compared to objective measures (polysomnography, PSG), in middle age and older individuals (Buysse et al., 2008, 1991). More recently, technological advances have led to the development of mobile activity

\footnotetext{
* Correspondence to: Bât. 2A - 2ème étage - Case 22, 146 rue Léo Saignat, 33076 Bordeaux cedex, France.

E-mail address: marion.baillet@u-bordeaux.fr (M. Baillet).
}

monitoring systems (actigraphy), a non-invasive technique easy to apply in large samples. Actigraphy has been used to objectively assess global activity/rest cycle including measures of daytime activity and sleep over a long-term period in a natural environment. Previous actigraphic studies have demonstrated that aging is not only related to sleep alterations but also to activity/rest cycle disturbances including fragmentation and a reduced amplitude of the cycle (Huang et al., 2002; van Someren et al., 1993). Evidence suggest that activity/rest cycle and sleep disruptions are related to cognitive deficits (Lim et al., 2013, 2012; Luik et al., 2015; Oosterman et al., 2009; Tranah et al., 2011; Walsh et al., 2014) and may represent earliest signs of neurodegenerative diseases (Wulff et al. 2010).

Although post-mortem and magnetic resonance imaging (MRI) investigations have reported widespread age-related changes in brain structure within grey (GM) and white matter (WM; Lockhart 
and DeCarli, 2014), few studies have evaluated associations between activity/rest cycle and brain modifications. GM atrophy, either widespread (Sexton et al., 2014) or circumscribed to the inferior prefrontal cortex (Branger et al., 2016; Lim et al., 2016), has been associated with disturbed sleep quality assessed through self-reported and actigraphic measures. Concerning the structural state of WM, research have shown that activity/rest cycle disturbances (fragmentation and reduction of amplitude) were related to WM integrity alterations assessed through WM hyperintensities (WMH; Oosterman et al., 2008; Zuurbier et al., 2015), suggesting an association between activity/rest cycle and cerebrovascular changes.

Diffusion Tensor Imaging (DTI) enables indirect investigation of WM microstructure via water diffusion within brain tissues and appears as a sensitive technique to describe age-related modifications of WM (Sullivan and Pfefferbaum, 2006). During aging, a consistent decrease of fractional anisotropy of water diffusion was described in the major WM tracts indicating a decrease of their anatomical coherence (Sullivan and Pfefferbaum, 2006). Moreover, recent research indicate that DTI parameters are modified before the appearance of $\mathrm{WMH}$ and therefore would reflect the early phase of WM changes (Maillard et al., 2013; Pelletier et al., 2015). Whereas age-related modifications of WM have been extensively investigated with DTI, relationship between DTI modifications and actigraphic parameters in aging have not been explored yet.

The present study aims to investigate associations of activity/ rest cycle assessed through actigraphy with GM atrophy and WM integrity in a population of elderly. Whole brain analyses will be conducted on GM volumes using voxel based morphometry pipeline and on WM integrity using tract based statistics analyses.

\section{Material and methods}

\subsection{Participants}

This study is a part of the AMImage research project, started in 2009 as an ancillary study of the AMI (Agrica-MSA-IFR de Santé Publique, Aging Multidisciplinary Investigation) cohort, an epidemiological prospective study on health and aging. The AMI cohort was composed of elderly people, aged $\geq 65$ years retired from agriculture and living in rural settings in South-West France. A more detailed description of the AMI cohort is provided elsewhere (Pérès et al., 2012). Study procedures were approved by the regional human research review board and all participants provided written informed consent (CHU de Bordeaux). At the second follow-up of AMImage, MRI sessions were followed by an actigraphic study performed on a sub-sample of participants $(n=91)$. Before analyses, all subjects were screened to exclude the following: major physical illness requiring treatment $(n=1$; leukemia), recordings containing less than 5 days $(n=5)$, sleep medications $(n=14)$, sleep complaints that had led to a diagnosis of sleep disorders $(n=5)$, external activity/rest cycle perturbations $(n=2)$, diagnosed dementia according to the National Institute of Neurological and Communication Disorders and Stroke/Alzheimer's Disease and Related Disorders Association (NINCDS-ADRDA) criteria $(n=1)$ and significant depressive symptomatology $(n=5$; CESD $>16$ ). A total of 58 elderly subjects were included in the present study.

\subsection{Demographic and clinical variables}

Participants' data were collected at home by a neuropsychologist to provide general information (age and gender) via questionnaires and to assess global cognitive functions using the Mini Mental State Examination (MMSE; Folstein et al., 1975) and depressive symptoms with the Center for Epidemiologic Studies Depression scale (CESD; Radloff, 1977).

Several clinical vascular risk factors were considered, including presence/absence of hypertension (blood pressure $>140 / 90 \mathrm{~mm}$ $\mathrm{Hg}$ ), of diabetes and of ischemic lesions on MRI images evaluated by a neuroradiologist.

\subsection{Activity/rest cycle assessment}

Activity/rest cycle was recorded by MotionWatch $8{ }^{\circledR}$ actigraph (Cambridge Neurotechnology, Cambridge, UK), equipped with a tri-axial accelerometer. This device was located at the nondominant wrist during a period of 7 days and 8 nights, in personal environment. Activity was measured in counts (i.e. acceleration episodes) on 60s epoch and data were analyzed with MotionWare $^{\circledR}$, version 1.0.3 (Cambridge Neurotechnology, Cambridge, UK).

A non-parametric approach was used to quantify the 24-h activity/rest cycle, more suitable than parametric analyses for the quantitative analysis of non-sinusoidal data. According to this type of analysis, three parameters were computed, the 24-h amplitude, the 24-h fragmentation and the 24-h stability (Van Someren et al., 1999). The 24-h amplitude represents the difference between the amount of activity occurring during the daytime period and the one occurring during the nighttime period. A high 24-h amplitude reflects a robust activity/rest cycle. The 24-h fragmentation quantifies the frequency and extent of transitions between activity and rest periods, i.e., the interruption of daytime activity by sleepiness periods and nighttime period by nocturnal arousals. A high 24-h fragmentation is often reported in pathological conditions (Hatfield et al., 2004). The 24-h stability evaluates the repetition of the 24-h activity/rest cycle pattern over 7 days, i.e., the strength of coupling of the activity/rest cycle to supposedly stable environmental synchronizer (Zeitgeber).

Considering sleep analysis, a sensitivity threshold of 40 counts was applied; a movement higher than 40 counts will be classified as active whereas a movement lower than 40 counts will be classified as rest. We reported total sleep time defined as the period between the onset and the offset of sleep minus the time classified as awake by the algorithm. To assess sleep quality, sleep fragmentation was used and calculated as the sum of the percentage of time spent in mobile periods and the percentage of immobile periods less or equal to one minute. Sleep parameters used in actigraphic studies were previously validated against PSG (Sadeh, 2011).

A minimum of 5 days of actigraphic recording was required for these variables to be reliably calculated.

\subsection{MRI acquisition}

MRI scans were obtained using an ACHIEVA 3T scanner (Philips Medical System, Netherlands) with a SENSE 8-channel head coil. Anatomical high resolution MRI volumes were acquired in transverse plan using a 3D MPRAGE T1-weighted sequence with the following parameters: $\mathrm{TR}=8.2 \mathrm{~ms}, \mathrm{TE}=3.5 \mathrm{~ms}$, 7-degree flip angle, FOV $256 \times 256 \mathrm{~mm}^{2}, 180$ slices, no gap and voxel size of $1 \times$ $1 \times 1 \mathrm{~mm}^{3}$. Two diffusion-weighted images (DTI) with opposite polarities were performed using a spin echo single shot EPI sequence with the following parameters: $\mathrm{TR}=6770 \mathrm{~ms}, \mathrm{TE}=60 \mathrm{~ms}$, 90-degree flip angle, FOV $224 \times 224 \mathrm{~mm}^{2}, 60$ slices, no gap and voxel size of $2 \times 2 \times 2 \mathrm{~mm}^{3}$. One b0 image was acquired and diffusion gradients were applied in 21 non-collinear directions (b-value $=1000 \mathrm{~s} / \mathrm{mm}^{2}$ ). To increase signal-to-noise ratio, the sequence was repeated in two successive runs for each polarity. All acquisitions were aligned on the anterior commissure-posterior commissure plan (AC-PC). Fluid-attenuated inversion recovery 
(FLAIR) images were also obtained with the following parameters: $\mathrm{TR}=11000 \mathrm{~ms}, \mathrm{TE}=140 \mathrm{~ms}, \mathrm{TI}=2800 \mathrm{~ms}$, 90-degree flip angle, FOV $230 \times 172 \mathrm{~mm}^{2}, 24$ slices of $5 \mathrm{~mm}$ of thickness, voxel size $0.72 \times 1.20 \times 5 \mathrm{~mm}^{3}$.

\subsection{MRI processing}

\subsubsection{Grey volume analysis}

Brain volumes were analyzed using the Voxel-Based Morphometry toolbox (VBM8; Ashburner and Friston, 2000) implemented in SPM8. Images were denoised and segmented into grey matter (GM), white matter (WM) and cerebro-spinal fluid (CSF) maps. These maps were warped to the Montreal Neurological Institute (MNI) space with a DARTEL type non-linear registration, modulated to preserve volume information and smoothed using a Gaussian kernel with a fullwidth half-maximum of $8 \mathrm{~mm}$. Total Intracranial Volume (TIV) was computed as the sum of the GM, WM and CSF volumes.

\subsubsection{White matter integrity analysis}

DTI images were pre-processed using FMRIB's Diffusion Toolbox in order to produce fractional anisotropy (FA), mean, axial and radial diffusivity (MD, AD and $\mathrm{RD}$ ) maps. The Tract Based Spatial Statistics (TBSS) pipeline was then applied on FA maps generated during the FDT pre-processing (Smith et al., 2006). A nonlinear registration was applied to align all subjects' FA maps to the FMRIB58_FA template. These maps were projected on a studyspecific mean FA skeleton template. This skeleton was created from 260 elderly subjects free of dementia and cerebral pathologies included at the first follow up of AMImage cohort. The same transformations were applied to $\mathrm{MD}, \mathrm{AD}$ and $\mathrm{RD}$ maps.

WMH were obtained by the lesion growth algorithm (Schmidt et al., 2012) as implemented in the Lesion Segmentation Tool (LST) toolbox version 1.1.7 for SPM8. Briefly, FLAIR images were co-registered to T1 images and using intensity distribution of FLAIR images, outliers were detected and lesion belief maps were calculated for each tissue classes (GM, WM and CSF). These maps were then summed up and a lesion growth model was applied to create lesion maps. WMH volumes were extracted and expressed as a percentage of TIV.

\subsection{Statistical analyses}

\subsubsection{Activity/rest cycle: relationship with demographic and clinical} variables

Because of the non-normality of measured variables distribution, we performed Spearman correlations to assess relationship between activity/rest cycle and continuous variables namely age, global cognition score, depressive symptoms score and WMH volumes. A Kruskal-Wallis test was used to measure associations of activity/rest cycle with categorical variables namely gender, presence/absence of hypertension, of diabetes and of ischemic lesions. Statistics were performed using the IBM $^{\circledR}{ }^{\circledR}$ SPSS ${ }^{\circledR}$ Statistics v.20 software (IBM Corporation, Armonk, NY, USA).

\subsubsection{Activity/rest cycle: relationship with GM volumes}

Whole brain analyses were performed using linear regressions with SPM8 to explore associations between activity/rest cycle and GM volumes extracted from VBM8 toolbox. Analyses were adjusted for age, gender and TIV. For all VBM analyses, we used a statistical threshold of $\mathrm{p}<0.05$ after corrections for multiple comparisons (False Discovery Rate, FDR) and a significant threshold cluster of 50 voxels.

\subsubsection{Activity/rest cycle: relationship with WM microstructure}

Linear regression models using FSL were computed to examine associations between activity/rest cycle and DTI indices. We first performed four models to describe associations of DTI indices (outcomes) with each activity/rest cycle parameters (predictors), controlling for age and gender. As 24-h amplitude includes measure of nighttime activity and consequently of sleep fragmentation (both parameters are significantly correlated), we then explored which 24-h amplitude or sleep was associated with WM integrity. For this, a fifth linear regression model was computed to test the association of DTI indices with 24-h amplitude (predictor), controlling for age, gender and sleep fragmentation. Finally, to investigate a potential impact of WMH burden, we added total WMH volumes as covariate in our multivariate model. Statistical inference was based on permutation-based statistics with 5000 permutations and threshold-free cluster enhancement (TFCE), with a threshold of $\mathrm{p}<0.05$ corrected for multiple comparisons. The WM atlas of the Johns Hopkins University was used to label significant results.

\section{Results}

From the initial cohort, 58 elderly with a mean age of 76 years were analyzed. Characteristics of participants and activity/rest cycle parameters are presented in Table 1.

\subsection{Activity/rest cycle: relationship with demographic and clinical variables}

Associations of demographic variables with activity/rest cycle (Table 2) revealed that an advanced age was associated with a lower 24-h amplitude $(\rho=-0.280, \mathrm{p}=0.033)$ and that men presented a 24 -h stability lower than women $(K=15.086, \mathrm{p}<0.001)$. Considering clinical factors, 24-h amplitude was negatively associated with WMH volumes $(\rho=-0.301, \mathrm{p}=0.022$; Table 2$)$ and a trend was observed for sleep fragmentation $(\rho=0.256 \mathrm{p}=0.052$ Table 2). None of the activity/rest cycle parameters were linked with global cognition or depressive symptoms scores, and other vascular risk factors (hypertension, diabetes and ischemic lesions).

Correlations analyses between activity/rest cycle parameters (Table 3 ) revealed that a less stable cycle (24-h stability) was associated with a higher 24 -h fragmentation $(\rho=-0.424, \mathrm{p}=0.001)$. A lower 24-h amplitude was related to a higher 24-h fragmentation $(\rho=-0.291, \mathrm{p}=0.027)$ and sleep fragmentation $(\rho=-0.664$, $\mathrm{p}<0.001)$.

Table 1

Characteristics of participants and activity/rest cycle parameters.

\begin{tabular}{ll}
\hline Variables & Mean \pm SEM or \% \\
\hline Demographic data & \\
Age (years) & $76.1 \pm 0.5$ \\
Female gender & $41 \%$ \\
Behavioral data & \\
MMSE score & $27.6 \pm 0.2$ \\
CESD score & $3.3 \pm 0.5$ \\
Vascular risk factors & \\
Hypertension & $64 \%$ \\
Diabetes & $5 \%$ \\
Ischemic lesions & $7 \%$ \\
WMH volumes (\% TIV) & $0.6 \pm 0.1$ \\
Activity/rest cycle parameters & \\
24-h amplitude & $0.92 \pm 0.005$ \\
24-h fragmentation & $0.77 \pm 0.026$ \\
24-h stability & $0.67 \pm 0.015$ \\
Sleep parameters & \\
Total sleep time & $07: 36 \pm 00: 05$ \\
Sleep fragmentation & $32.42 \pm 1.481$ \\
\hline
\end{tabular}


Table 2

Relationship of activity/rest cycle with demographic and clinical variables.

\begin{tabular}{|c|c|c|c|c|}
\hline & $\begin{array}{l}\text { 24-h Am- } \\
\text { plitude } \\
\rho^{\mathrm{a}} \text { or } \mathrm{K}^{\mathrm{b}}\end{array}$ & $\begin{array}{l}\text { 24-h Frag- } \\
\text { mentation } \\
\rho^{\mathrm{a}} \text { or } \mathrm{K}^{\mathrm{b}}\end{array}$ & $\begin{array}{l}\text { 24-h Stability } \\
\rho^{\mathrm{a}} \text { or } \mathbf{K}^{\mathrm{b}}\end{array}$ & $\begin{array}{l}\text { Sleep Frag- } \\
\text { mentation } \\
\rho^{\mathrm{a}} \text { or } \mathrm{K}^{\mathrm{b}}\end{array}$ \\
\hline Age $^{a}$ & $-0.280^{*}$ & 0.184 & -0.245 & 0.140 \\
\hline Gender $^{\mathrm{b}}$ & 0.389 & 0.587 & $15.086^{* * * * * *}$ & 0.440 \\
\hline MMSE $^{\mathrm{a}}$ & 0.110 & -0.124 & 0.088 & -0.149 \\
\hline $\mathrm{CESD}^{\mathrm{a}}$ & -0.187 & -0.009 & 0.120 & 0.101 \\
\hline Hypertension $^{\mathrm{b}}$ & 0.213 & 0.041 & 0.005 & 0.654 \\
\hline Diabetes $^{\mathrm{b}}$ & 0.100 & 2.784 & 1.223 & 0.178 \\
\hline $\begin{array}{l}\text { Ischemic } \\
\text { lesions }^{\mathrm{b}}\end{array}$ & 0.477 & 0.015 & 0.015 & 0.377 \\
\hline $\begin{array}{l}\text { WMH vo- } \\
\text { lumes (\% } \\
\text { TIV) })^{\mathrm{a}}\end{array}$ & $-0.301^{*}$ & 0.012 & -0.141 & 0.256 \\
\hline
\end{tabular}

a Spearman correlation $\rho$

${ }^{\mathrm{b}}$ Kruskal Wallis test $\mathrm{K}$;

$* \mathrm{p}<0.05$,

$* * * * \mathrm{p}<0.001$

Table 3

Relationship between activity/rest cycle parameters.

\begin{tabular}{llll}
\hline & 24-h Amplitude & 24-h Fragmentation & 24-h Stability \\
& $\boldsymbol{\rho}$ & $\boldsymbol{\rho}$ & $\boldsymbol{\rho}$ \\
\hline 24-h Amplitude & - & - & - \\
24-h Fragmentation & $-0.291^{*}$ & - & - \\
24-h Stability & 0.156 & $-0.424^{* * * *}$ & - \\
Sleep Fragmentation & $-0.664^{* * * *}$ & 0.061 & 0.083 \\
\hline
\end{tabular}

Spearman correlation $\rho$;

$* \mathrm{p}<0.05$,

$* * * * \mathrm{p}<0.001$.

\subsection{Activity/rest cycle: relationship with GM volume}

No significant association was found between GM volumes of brain area and activity/rest cycle parameters.

\subsection{Activity/rest cycle: relationship with WM microstructure}

TBSS analyses showed significant associations between DTI indices and 24-h amplitude ( $\mathrm{p}<0.05$, TFCE corrected; Fig. 1). A diminution of 24-h amplitude was associated with lower FA values and higher diffusivity values (MD and RD) in mostly WM areas, including the corpus callosum, external and internal capsule and the fornix. No significant result was found with 24-h fragmentation or 24-h stability.

A significant association was also observed between DTI indices and sleep fragmentation. An increase of sleep fragmentation was related to a diminution of FA values ( $p<0.05$, TFCE corrected; Fig. 2). Less extended results were found with $R D$ values $(p<0.05$, TFCE corrected; Fig. 2) and with MD values which remained under the significant threshold ( $\mathrm{p}=0.054$, TFCE corrected).

In a model adjusted for age, gender and sleep fragmentation, the association of 24-h amplitude with FA and RD decreased but remained significant in several WM pathways (MD did not reach significant threshold $\mathrm{p}=0.053$, TFCE corrected).

In the last model adjusted for age, gender and WMH volumes, the association between 24-h amplitude and DTI indices did not reach significant threshold after multiple comparisons adjustment. As shown in Fig. A.1, 4 outliers of WMH were present in our sample; while most of participants had a WMH volumes between 0 and $1.5 \%$ of TIV $(0-20 \mathrm{~mL}$ respectively), these subjects presented $\mathrm{WMH}$ volumes ranging from $2.3 \%$ to $3.7 \%$ of TIV $(30-52 \mathrm{~mL}$ respectively). When those were excluded from regression analysis, the association between 24-h amplitude and DTI indices (FA, RD and $\mathrm{MD}$ ) remained significant after adjusting by WMH volumes in several WM areas (Fig. A.2).

\section{Discussion}

In this study of 58 older persons, a lower 24-h amplitude and a higher sleep fragmentation were both associated with WM microstructure alterations, independently of age and gender whereas no association was observed with GM volumes. In addition, our statistical analyses indicated that not only sleep but also 24-h cycle were associated with WM integrity in aging subjects.

Compared to other studies on elderly, we observed a higher 24h amplitude, a higher 24-h stability and a lower 24-h fragmentation suggesting that our population was relatively preserved for activity/rest cycle (Hatfield et al., 2004; Huang et al., 2002). A higher sleep duration and quality were also observed (Huang et al., 2002). Subjects included in our study were free of diagnosed sleep disorders and sleep medication explaining their relative preservation of activity/rest cycle (Dawson et al., 2008; Sadeh et al., 1995). Despite this preservation and in accordance with the literature, we found that men presented a 24-h stability lower than women (Luik et al., 2013) and we observed an age-related alteration of the 24-h amplitude. In contrast to other studies (Huang et al., 2002; Luik et al., 2013), no age effect was observed on the 24-h fragmentation, 24-h stability and sleep fragmentation. However, this is consistent with studies suggesting that reduced 24-h amplitude is the most prominent activity/rest cycle changes in aging (Huang et al., 2002; van Someren et al., 1993). As expected, we reported an inverse correlation between 24-h stability and 24-h fragmentation (Luik et al., 2013; Zuurbier et al., 2015), but also an association between 24-h amplitude and 24-h fragmentation (Luik et al., 2013). In our population, 24-h fragmentation of the activity/rest cycle was not associated with sleep fragmentation. This suggests that 24 -h fragmentation in our population of elderly would rather be related to the fragmentation of the active period than to the one of the rest period. However, the association of these two parameters should be considered with caution since these parameters were computed on different time scales; short sleep perturbations during one or several minutes might weakly impact the 24 -h fragmentation parameter.

In our population of 58 community-dwelling elderly, we found an association between 24-h amplitude of the activity/rest cycle and a widespread alteration of WM integrity, whenever WMH are taking into account. Even if 24-h amplitude and sleep fragmentation are related to WM integrity disruption, results from the model accounted for sleep fragmentation suggest that not only sleep but also 24-h cycle are associated with WM integrity. Some evidence in the literature showed that physical activity influences both vascular health (Lacey et al., 2015) and WM integrity (Tseng et al., 2013). Physical activity could therefore account for a part of the association between 24-h amplitude and WM integrity observed in our population. Since actigraphy provides a measure of mean daily activity - an indirect measure of physical activity - we could test this hypothesis. In our population, no significant association was found between mean daily activity and vascular risk factors (presence/absence of hypertension, of diabetes, of ischemic lesions and WMH volumes; data not shown). Concerning WM integrity, the association between 24-h amplitude and DTI parameters (FA, MD and RD) remained significant ( $p<0.05$, TFCE corrected) in a model accounting for age, gender and mean daily activity (data not shown). This set of analyses suggests that the association between the activity/rest cycle and WM integrity is not driven by the amount of daily activity in our population. Moreover, 


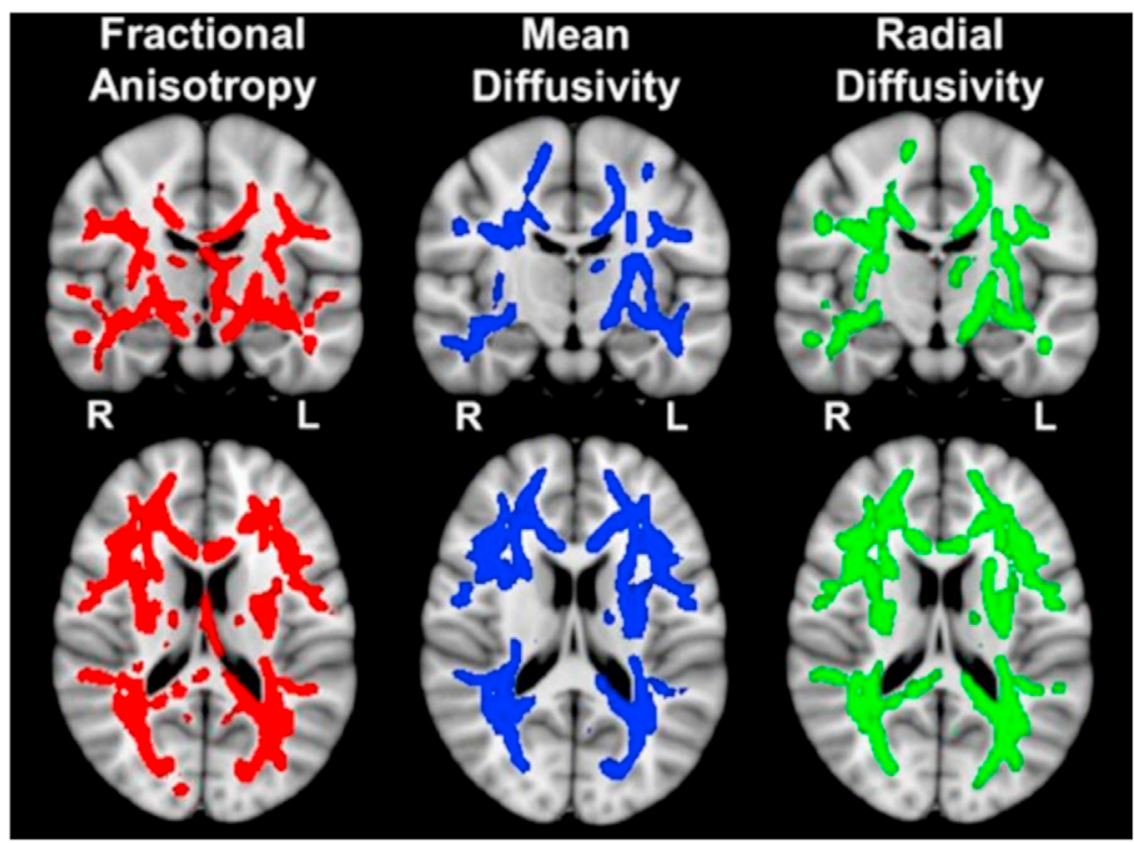

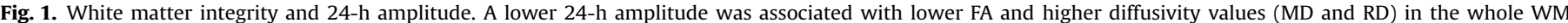
skeleton after adjustment for age and gender. Results were displayed at $\mathrm{p}<0.05$, TFCE corrected and overlaid on the MNI template.

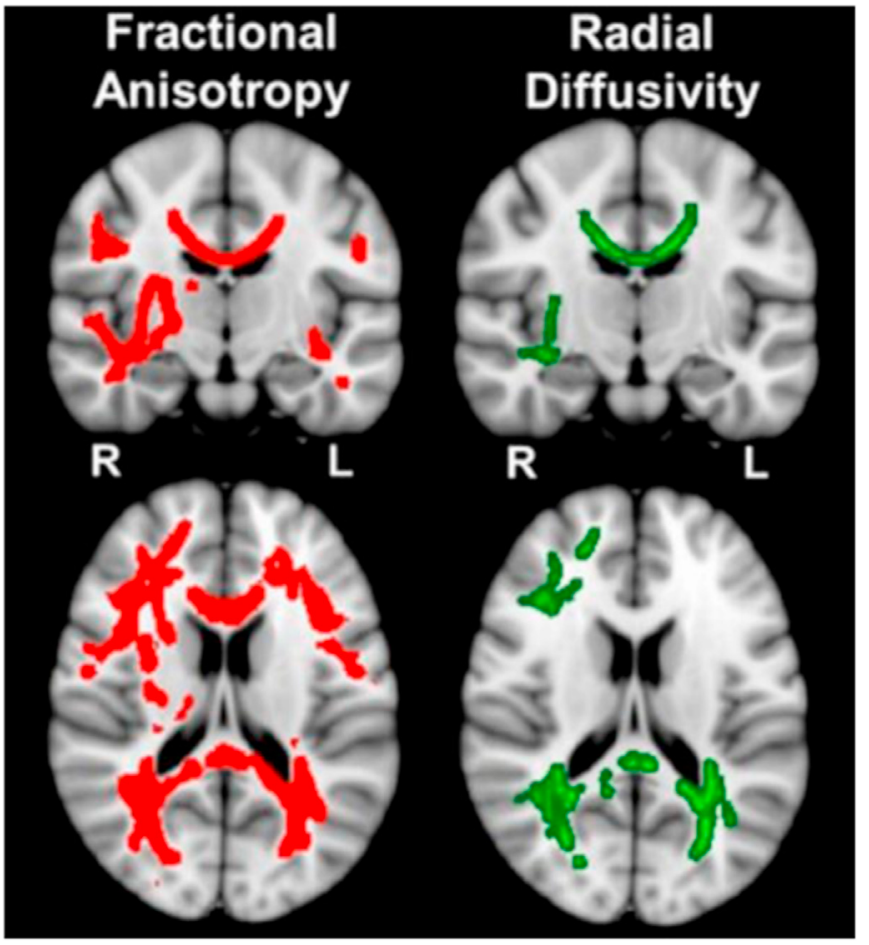

Fig. 2. White matter integrity and sleep fragmentation. A higher sleep fragmentation was associated with lower FA and higher RD values after adjustment for age and gender. Results were displayed at $\mathrm{p}<0.05$, TFCE corrected and overlaid on the MNI template.

regular physical practice has been shown to influence sleep quality (Youngstedt, 2005). No significant correlation between mean daily activity and sleep fragmentation was found in our population (data not shown). Noteworthy, the amount of activity measured by actigraphy does not indicate neither the intensity of physical activity nor the type of activity (aerobic or anaerobic) - both pertinent parameters of physical activity (Nelson et al., 2007).

These results highlight important findings, as they provide evidence that 24-h amplitude alterations are associated with cerebral frailty mainly underpinned by an alteration of brain structural connectivity. Considering the relationship between cerebral WM integrity alterations and cognition (Lockhart and DeCarli, 2014), our results suggest a neuroanatomical substrate explaining the risk of cognitive impairment or dementia associated with activity/rest cycle perturbations (Lim et al., 2013, 2012; Luik et al. 2015; Oosterman et al., 2009; Tranah et al., 2011; Walsh et al., 2014).

We observed an association between 24-h amplitude and DTI indices partly impacted by WMH burden. This is in accordance with previous studies showing a significant association between disrupted activity/rest cycle and the presence of WMH (Oosterman et al., 2008; Zuurbier et al., 2015). We also observed that a lower 24-h amplitude is related to altered DTI parameters not only in regions presenting WMH (periventricular and deep WM regions) but also in peripheral normal-appearing WM. Moreover, after exclusion of 4 outliers (high volumes of $\mathrm{WMH}$ ), the association remained significant after correction by WMH volumes. Regarding these results, it could be hypothesized that a diminution of 24-h amplitude might be associated with a global alteration of WM microstructure independently of WMH burden. WMH and DTI parameters modifications are both agerelated changes observed in MRI studies (Lockhart and DeCarli, 2014). Recent studies indicate that DTI modifications and the presence of $\mathrm{WMH}$ are two interdependent processes occurring within different temporal windows; in that DTI changes are part of a continuum of WM degeneration with WMH being the ultimate phase (Maillard et al., 2013; Pelletier et al., 2015). Even if physiological interpretations of DTI parameters should be drawn with cautious (Wheeler-Kingshott and Cercignani, 2009), the increase of RD could reflect a dysmyelination rather than a loss of axonal integrity driven by AD modifications (Song et al., 2002). Since WMH in elderly have a vascular origin, our results suggest that cerebral frailty associated with age-related activity/rest cycle dysfunction has a sub-clinical vascular origin. Consistently, some studies have suggested that disrupted activity/rest cycle in older adults, particularly reduced 24-h amplitude measured by actigraphy, was associated with increased risk of cardiovascular disease and mortality (Paudel et al., 2011).

We did not find any association between activity/rest cycle and 
GM volumes. Age-related atrophy and sleep fragmentation were sparsely investigated and inconsistent results were obtained in three previous MRI studies (Branger et al., 2016; Lim et al., 2016; Sexton et al., 2014). For one of them, sleep quality was related to widespread GM atrophy (Sexton et al., 2014) whereas for the others, sleep quality was associated with regional brain atrophy (Branger et al., 2016; Lim et al., 2016). Methodological considerations could explain these discrepancies; in two previous studies (Branger et al., 2016; Sexton et al., 2014), sleep quality was evaluated through the Pittsburg Sleep Quality Index (Buysse et al., 1989). Considering the relative inaccuracy of these evaluations compared to objective measures in healthy individuals (Buysse et al., 2008, 1991; Grandner et al., 2006; Landry et al., 2015), results from these studies might be difficult to compare with those using actigraphy or PSG. In Lim study (Lim et al., 2016), a higher sample size, a different algorithm of segmentation (cortical thickness parameters through FreeSurfer analysis) and a more sensitive parameter ( 0 count as a threshold for classification of activity or rest period) might explain their higher sensitivity for GM atrophy. Moreover, subjects with diagnosed sleep disorders were not excluded from their analyses. Considering that pathological sleep situations like insomnia (Altena et al., 2010) or sleep apnea (Torelli et al., 2011) are reported to be associated with both sleep fragmentation and neuroanatomical changes especially in frontal area, these types of disorders might contribute to their results.

Methodological limitations have to be considered in interpreting our data. Firstly, this cross-sectional study was not designed to explore the causal association between activity/rest cycle and brain structure alterations. Despite its widespread use, TBSS pipeline and its skeleton approach is still questionable regarding the confining statistical testing to voxels within the skeleton $(\mathrm{Za}-$ lesky, 2011) and the possible loss of anatomical consistency (de Groot et al., 2013). Secondly as in most actigraphic studies, individuals with undiagnosed sleep-disordered breathing in our population cannot be excluded. Finally, we did not find significant association between activity/rest cycle parameters and global cognitive status evaluated by using MMSE, which might not be sensitive enough to detect subtle inter-individual differences in healthy older adults. Another potential limitation is the generalizability of our results to other populations for two reasons. Firstly, this study was based on a small sample size of healthy individuals who accepted to participate in both MRI and actigraphic studies. Despite this selection bias, our population exhibited sufficient inter-individual variability in activity/rest cycle and WM integrity to detect associations between these parameters. Secondly, the cohort is composed by retired farmers living in rural settings, limiting the comparison with other populations living in urban areas. Facing these considerations, our study presents several strengths. To our knowledge, this is one of the first study assessing associations of activity/rest cycle through actigraphy with both measures of GM atrophy and WM integrity in a population of older individuals. All potential effects of comorbid conditions were limited with the exclusion of diagnosed sleep disorders, sleep medications (Dawson et al., 2008) and prominent depressive symptoms (Alvaro et al., 2013). We believe that further studies are needed to confirm our results in a larger cohort of elderly people.

\section{Conclusion}

This study showed that in our population of elderly persons, a diminution of 24-h amplitude of the activity/rest cycle was associated with a cerebral frailty of structural connectivity and suggests a neuroanatomical substrate to explain the association between activity/rest cycle and cognition. While the majority of studies focused on sleep, this one highlights the importance of activity/rest cycle on brain structure. Considering that during aging WM microstructure alterations are often related to vascular risk factors, further studies are required to examine their links with activity/rest cycle disruptions and temporal sequences of these events.

\section{Author disclosures}

M.B., B.D., K.P., W.M. and G.C. report no disclosures. Jean-François Dartigues reports grants from IPSEN and Roche.

\section{Study funding}

The AMI project was funded by AGRICA (CAMARCA, CRCCA, CCPMA PREVOYANCE, CPCEA, AGRI PREVOYANCE), Mutualité Sociale Agricole (MSA) de Gironde, Caisse Centrale de la Mutualité Sociale Agricole (CCMSA) and Caisse Nationale pour la Solidarité et l'Autonomie (CNSA). The AMImage2 project was supported by grants from DGOS (Direction Générale de l'Offre de Soins). This study was achieved within the context of the Laboratory of Excellence TRAIL ANR-10-LABX-57.

\section{Acknowledgements}

The authors thank the participants and the staff of the AMI project and Caroline Buisson for her technical assistance.

\section{Appendix A. Supporting information}

Supplementary data associated with this article can be found in the online version at http://dx.doi.org/10.1016/j.neuroimage.2016.09.051.

\section{References}

Altena, E., Vrenken, H., Van Der Werf, Y.D., van den Heuvel, O.A., Van Someren, E.J W., 2010. Reduced orbitofrontal and parietal gray matter in chronic insomnia: a voxel-based morphometric study. Biol. Psychiatry 67, 182-185. http://dx.doi. org/10.1016/j.biopsych.2009.08.003.

Alvaro, P.K., Roberts, R.M., Harris, J.K., 2013. A systematic review assessing bidirectionality between sleep disturbances, anxiety, and depression. Sleep 36 1059-1068. http://dx.doi.org/10.5665/sleep.2810.

Ashburner, J., Friston, K.J., 2000. Voxel-based morphometry-the methods. NeuroImage 11, 805-821. http://dx.doi.org/10.1006/nimg.2000.0582.

Branger, P., Arenaza-Urquijo, E.M., Tomadesso, C., Mézenge, F., André, C., Flores, R., de, Mutlu, J., Sayette, V., de, L., Eustache, F., Chételat, G., Rauchs, G., 2016. Relationships between sleep quality and brain volume, metabolism, and amyloid deposition in late adulthood. Neurobiol. Aging 41, 107-114. http://dx.doi.org/ 10.1016/j.neurobiolaging.2016.02.009.

Buysse, D.J., Hall, M.L., Strollo, P.J., Kamarck, T.W., Owens, J., Lee, L., Reis, S.E., Matthews, K A., 2008. Relationships between the Pittsburgh Sleep Quality Index (PSOI), Epworth Sleepiness Scale (ESS), and clinical/polysomnographic measures in a community sample. J. Clin. Sleep. Med. JCSM Publ. Am. Acad. Sleep. Med. 4, 563-571.

Buysse, D.J., Reynolds, C.F., Monk, T.H., Berman, S.R., Kupfer, D.J., 1989. The Pittsburgh Sleep Quality Index: a new instrument for psychiatric practice and research. Psychiatry Res 28, 193-213.

Buysse, D.J., Reynolds, C.F., Monk, T.H., Hoch, C.C., Yeager, A.L., Kupfer, D.J., 1991. Quantification of subjective sleep quality in healthy elderly men and women using the Pittsburgh Sleep Quality Index (PSQI). Sleep 14, 331-338.

Dawson, J., Boyle, J., Stanley, N., Johnsen, S., Hindmarch, I., Skene, D.J., 2008. Benzodiazepine-induced reduction in activity mirrors decrements in cognitive and psychomotor performance. Hum. Psychopharmacol. 23, 605-613. http://dx.doi. org/10.1002/hup.961.

de Groot, M., Vernooij, M.W., Klein, S., Ikram, M.A., Vos, F.M., Smith, S.M., Niessen, W.J., Andersson, J.L.R., 2013. Improving alignment in Tract-based spatial statistics: evaluation and optimization of image registration. Neurolmage 76, 400-411. http://dx.doi.org/10.1016/j.neuroimage.2013.03.015.

Folstein, M.F., Folstein, S.E., McHugh, P.R., 1975. "Mini-mental state". A practical method for grading the cognitive state of patients for the clinician. J. Psychiatry Res. 12, 189-198. Grandner, M.A., Kripke, D.F., Yoon, I.-Y., Youngstedt, S.D., 2006. Criterion validity of the Pittsburgh Sleep Quality Index: investigation in a non-clinical sample. Sleep. Biol. Rhythm. 4, 129-139. http://dx.doi.org/10.1111/j.1479-8425.2006.00207.x. 
Hatfield, C.F., Herbert, J., van Someren, E.J.W., Hodges, J.R., Hastings, M.H., 2004. Disrupted daily activity/rest cycles in relation to daily cortisol rhythms of home-dwelling patients with early Alzheimer's dementia. Brain. J. Neurol. 127, 1061-1074. http://dx.doi.org/10.1093/brain/awh129.

Huang, Y.-L., Liu, R.-Y., Wang, Q.-S., Van Someren, E.J.W., Xu, H., Zhou, J.-N., 2002 Age-associated difference in circadian sleep-wake and rest-activity rhythms. Physiol. Behav. 76, 597-603.

Johns, M.W., 1991. A new method for measuring daytime sleepiness: the Epworth sleepiness scale. Sleep 14, 540-545.

Lacey, B., Golledge, J., Yeap, B.B., Lewington, S., Norman, P.E., Flicker, L., Almeida, O. P., Hankey, G.J., 2015. Physical activity and vascular disease in a prospective cohort study of older men: the Health In Men Study (HIMS). BMC Geriatr. 15, 164. http://dx.doi.org/10.1186/s12877-015-0157-2.

Landry, G.J., Best, J.R., Liu-Ambrose, T., 2015. Measuring sleep quality in older adults: a comparison using subjective and objective methods. Front. Aging Neurosci. 7, 166. http://dx.doi.org/10.3389/fnagi.2015.00166.

Lim, A.S.P., Fleischman, D.A., Dawe, R.J., Yu, L., Arfanakis, K., Buchman, A.S., Bennett, D.A., 2016. Regional neocortical gray matter structure and sleep fragmentation in older adults. Sleep 39, 227-235. http://dx.doi.org/10.5665/sleep.5354.

Lim, A.S.P., Kowgier, M., Yu, L., Buchman, A.S., Bennett, D.A., 2013. Sleep fragmentation and the risk of incident alzheimer's disease and cognitive decline in older persons. Sleep 36, 1027-1032. http://dx.doi.org/10.5665/sleep.2802.

Lim, A.S.P., Yu, L., Costa, M.D., Leurgans, S.E., Buchman, A.S., Bennett, D.A., Saper, C. B., 2012. Increased fragmentation of rest-activity patterns is associated with a characteristic pattern of cognitive impairment in older individuals. Sleep 35, 633-640B. http://dx.doi.org/10.5665/sleep.1820.

Lockhart, S.N., DeCarli, C., 2014. Structural imaging measures of brain aging. Neuropsychol. Rev. 24, 271-289. http://dx.doi.org/10.1007/s11065-014-9268-3.

Luik, A.I., Zuurbier, L.A., Hofman, A., Van Someren, E.J.W., Ikram, M.A., Tiemeier, H., 2015. Associations of the 24-h activity rhythm and sleep with cognition: a population-based study of middle-aged and elderly persons. Sleep. Med. 16, 850-855. http://dx.doi.org/10.1016/j.sleep.2015.03.012.

Luik, A.I., Zuurbier, L.A., Hofman, A., Van Someren, E.J.W., Tiemeier, H., 2013. Stability and fragmentation of the activity rhythm across the sleep-wake cycle: the importance of age, lifestyle, and mental health. Chronobiol. Int. 30, 1223-1230. http://dx.doi.org/10.3109/07420528.2013.813528.

Maillard, P., Carmichael, O., Harvey, D., Fletcher, E., Reed, B., Mungas, D., DeCarli, C., 2013. FLAIR and diffusion MRI signals are independent predictors of white matter hyperintensities. AJNR Am. J. Neuroradiol. 34, 54-61. http://dx.doi.org/ 10.3174/ajnr.A3146.

Nelson, M., Rejeski, W., Blair, S., Duncan, P., Judge, J., King, A., Macera, C., CastanedaSceppa, C., 2007. Physical activity and public health in older adults: recommendation from the American college of sports medicine and the American Heart Association. Circulation 116, 1094-1105.

Oosterman, J., van Harten, B., Vogels, R., Gouw, A., Weinstein, H., Scheltens, P. Scherder, E., 2008. Distortions in rest-activity rhythm in aging relate to white matter hyperintensities. Neurobiol. Aging 29, 1265-1271. http://dx.doi.org/ 10.1016/j.neurobiolaging.2007.02.014.

Oosterman, J.M., van Someren, E.J.W., Vogels, R.L.C., Van Harten, B., Scherder, E.J.A., 2009. Fragmentation of the rest-activity rhythm correlates with age-related cognitive deficits. J. Sleep. Res. 18, 129-135. http://dx.doi.org/10.1111/ j.1365-2869.2008.00704.x.

Paudel, M.L., Taylor, B.C., Ancoli-Israel, S., Stone, K.L., Tranah, G., Redline, S., BarrettConnor, E., Stefanick, M.L., Ensrud, K.E., 2011. Rest/activity rhythms and cardiovascular disease in older men. Chronobiol. Int. 28, 258-266. http://dx.doi.org/ 10.3109/07420528.2011.553016.

Pelletier, A., Periot, O., Dilharreguy, B., Hiba, B., Bordessoules, M., Chanraud, S., Pérès, K., Amieva, H., Dartigues, J.-F., Allard, M., Catheline, G., 2015. Age-related modifications of diffusion tensor imaging parameters and white matter hyperintensities as inter-dependent processes. Front. Aging Neurosci. 7, 255. http: //dx.doi.org/10.3389/fnagi.2015.00255.

Pérès, K., Matharan, F., Allard, M., Amieva, H., Baldi, I., Barberger-Gateau, P., Bergua, V. Bourdel-Marchasson, I., Delcourt, C., Foubert-Samier, A., Fourrier-Réglat, A., Gaimard, M., Laberon, S., Maubaret, C., Postal, V., Chantal, C., Rainfray, M., Rascle, N., Dartigues, J.-F., 2012. Health and aging in elderly farmers: the AMI cohort. BMC Public Health 12, 558. http://dx.doi.org/10.1186/1471-2458-12-558.
Radloff, L.S., 1977. The CES-D scale a self-report depression scale for research in the general population. Appl. Psychol. Meas. 1, 385-401. http://dx.doi.org/10.1177/ 014662167700100306.

Sadeh, A., 2011. The role and validity of actigraphy in sleep medicine: an update. Sleep. Med. Rev. 15, 259-267. http://dx.doi.org/10.1016/j.smrv.2010.10.001.

Sadeh, A., Hauri, P.J., Kripke, D.F., Lavie, P., 1995. The role of actigraphy in the evaluation of sleep disorders. Sleep 18, 288-302.

Schmidt, P., Gaser, C., Arsic, M., Buck, D., Förschler, A., Berthele, A., Hoshi, M., Ilg, R. Schmid, V.J., Zimmer, C., Hemmer, B., Mühlau, M., 2012. An automated tool for detection of FLAIR-hyperintense white-matter lesions in Multiple Sclerosis. NeuroImage 59, 3774-3783. http://dx.doi.org/10.1016/j.neuroimage.2011.11.032.

Sexton, C.E., Storsve, A.B., Walhovd, K.B., Johansen-Berg, H., Fjell, A.M., 2014. Poor sleep quality is associated with increased cortical atrophy in community-dwelling adults. Neurology 83, 967-973. http://dx.doi.org/10.1212/ WNL.0000000000000774.

Smith, S.M., Jenkinson, M., Johansen-Berg, H., Rueckert, D., Nichols, T.E., Mackay, C.E. Watkins, K.E., Ciccarelli, O., Cader, M.Z., Matthews, P.M., Behrens, T.E.J., 2006. Tract-based spatial statistics: voxelwise analysis of multi-subject diffusion data NeuroImage 31, 1487-1505. http://dx.doi.org/10.1016/j.neuroimage.2006.02.024.

Song, S.-K., Sun, S.-W., Ramsbottom, M.J., Chang, C., Russell, J., Cross, A.H., 2002. Dysmyelination revealed through MRI as increased radial (but unchanged axial) diffusion of water. NeuroImage 17, 1429-1436.

Sullivan, E.V., Pfefferbaum, A., 2006. Diffusion tensor imaging and aging. Neurosci. Biobehav. Rev., Methodological and Conceptual Advances in the Study of BrainBehavior Dynamics: A Multivariate Lifespan Perspective 30, pp. 749-761. doi:10.1016/j.neubiorev.2006.06.002.

Torelli, F., Moscufo, N., Garreffa, G., Placidi, F., Romigi, A., Zannino, S., Bozzali, M., Fasano, F., Giulietti, G., Djonlagic, I., Malhotra, A., Marciani, M.G., Guttmann, C.R., 2011 Cognitive profile and brain morphological changes in obstructive sleep apnea. Neurolmage 54, 787-793. http://dx.doi.org/10.1016/j.neuroimage.2010.09.065.

Tranah, G.J., Blackwell, T., Stone, K.L., Ancoli-Israel, S., Paudel, M.L., Ensrud, K.E. Cauley, J.A., Redline, S., Hillier, T.A., Cummings, S.R., Yaffe, K., Research Group, S. O.F., 2011. Circadian activity rhythms and risk of incident dementia and mild cognitive impairment in older women. Ann. Neurol. 70, 722-732. http://dx.doi. org/10.1002/ana.22468.

Tseng, B.Y., Gundapuneedi, T., Khan, M.A., Diaz-Arrastia, R., Levine, B.D., Lu, H., Huang, H., Zhang, R., 2013. White matter integrity in physically fit older adults. NeuroImage 0, 510-516. http://dx.doi.org/10.1016/j.neuroimage.2013.06.011.

van Someren, E.J., Mirmiran, M., Swaab, D.F., 1993. Non-pharmacological treatment of sleep and wake disturbances in aging and Alzheimer's disease: chronobiological perspectives. Behav. Brain Res. 57, 235-253.

Van Someren, E.J., Swaab, D.F., Colenda, C.C., Cohen, W., McCall, W.V., Rosenquist, P. B., 1999. Bright light therapy: improved sensitivity to its effects on rest-activity rhythms in Alzheimer patients by application of nonparametric methods. Chronobiol. Int. 16, 505-518.

Walsh, C.M., Blackwell, T., Tranah, G.J., Stone, K.L., Ancoli-Israel, S., Redline, S., Paudel, M., Kramer, J.H., Yaffe, K., 2014. Weaker circadian activity rhythms are associated with poorer executive function in older women. Sleep 37, 2009-2016. http://dx.doi.org/10.5665/sleep.4260.

Wheeler-Kingshott, C.A.M., Cercignani, M., 2009. About "axial" and "radial" diffusivities. Magn. Reson. Med. 61, 1255-1260. http://dx.doi.org/10.1002/mrm.21965.

Wulff, K., Gatti, S., Wettstein, J.G., Foster, R.G., 2010. Sleep and circadian rhythm disruption in psychiatric and neurodegenerative disease. Nat. Rev. Neurosci. 11 589-599. http://dx.doi.org/10.1038/nrn2868.

Youngstedt, S.D., 2005. Effects of exercise on sleep. Clin. Sport. Med. Sport. Chrono-: Time 24, 355-365. http://dx.doi.org/10.1016/j.csm.2004.12.003.

Zalesky, A., 2011. Moderating registration misalignment in voxelwise comparisons of DTI data: a performance evaluation of skeleton projection. Magn. Reson. Imaging 29, 111-125. http://dx.doi.org/10.1016/j.mri.2010.06.027.

Zuurbier, L.A., Ikram, M.A., Luik, A.I., Hofman, A., Van Someren, E.J.W., Vernooij, M W., Tiemeier, H., 2015. Cerebral small vessel disease is related to disturbed 24-h activity rhythms: a population-based study. Eur. J. Neurol. 22, 1482-1487. http: //dx.doi.org/10.1111/ene.12775. 